\title{
Primary adrenal schwannoma with catecholamine hypersecretion
}

Jianglong Hou' ${ }^{1}$, Lizhi Zhang ${ }^{2}$, Yingkun Guo ${ }^{3}$, Huizhu Chen ${ }^{3}$, Weiya Wang ${ }^{4}$

${ }^{1}$ Department of Thoracic and Cardiovascular Surgery, West China Hospital, Sichuan University, Sichuan, China

2Department of Radiology, West China Hospital, Sichuan University, Sichuan, China ${ }^{3}$ Department of Radiology, West China Second University Hospital, Sichuan University, Sichuan, China

${ }^{4}$ Department of Pathology, West China Hospital, Sichuan University, Sichuan, China

Submitted: 4 July 2014

Accepted: 27 September 2014

Arch Med Sci 2016; 12, 3: 681-683

DOI: 10.5114/aoms.2016.59942

Copyright @ 2016 Termedia \& Banach

Schwannomas are benign tumors of the nerve sheath Schwann cell or nerve cell sheath, so the tumor is also called neurilemmoma. Although this tumor can involve any place where Schwann cells are present, it arises predominantly in the head and neck region or the flexor surfaces of the extremities. Schwannomas may also occur in the retroperitoneum, and they account for approximately $5 \%$ of the primary neoplasms in this area [1]. However, primary schwannomas of the adrenal gland are extremely rare. Primary adrenal schwannomas occur most often as a rare type of incidentaloma with no evidence of hormonal hypersecretion [18]. When adrenal neoplasia with catecholamine hypersecretion is found, pheochromocytomas/paragangliomas need be differentiated. If pheochromocytomas/paragangliomas are overlooked or improperly treated, the clinical outcomes almost invariably prove fatal [9]. However, this case is of interest in that schwannoma arising from the adrenal gland with catecholamine hypersecretion, and its clinical presence and imaging features, must be carefully evaluated and further diagnosed.

A 41-year-old woman presented with 2 months history of left upper quadrant abdominal discomfort. Her past medical or surgical history was not significant. Physical examination revealed left upper quadrant and left flank tenderness on deep palpation, and no palpable masses were identified. The results of hormonal laboratory tests found that cortisol levels were elevated (adrenaline $569 \mathrm{ng} / \mathrm{l}$, norepinephrine $483 \mathrm{ng} / \mathrm{l}$ ). Serum aldosterone, electrolytes, urinary metanephrine, and vanillylmandelic acid were within the normal range. Multi-detector computed tomography (MDCT) examination detected a well-defined mass in the left adrenal gland, measuring approximately $7.7 \times 6.4 \times 7.1 \mathrm{~cm}$. Computed tomography (CT) images showed heterogeneous density of the mass with dot-like calcification (Figure 1). The left kidney and pancreas in the vicinity were compressed. No sign of bony or visceral involvement was revealed. Local excision was performed under anesthesia. A well-demarcated tumor in the left adrenal gland was identified and entirely removed without complication. Histopathologic examination confirmed that the tumor exhibited Antoni A areas composed of well-defined fascicles of spindle cells and loosely organized Antoni B areas. Immunohistochemical staining demonstrated that the tumor was positive for S-100 protein (Figure 2). Based on these findings, the final diagnosis was benign

\author{
Corresponding author: \\ Yingkun Guo MD \\ Department of Radiology \\ West China Second \\ University Hospital \\ Sichuan University \\ 20\# South \\ Renmin Road \\ 610041 Sichuan, China \\ Phone: +8628 855503275(0) \\ E-mail: gykpanda@163.com
}



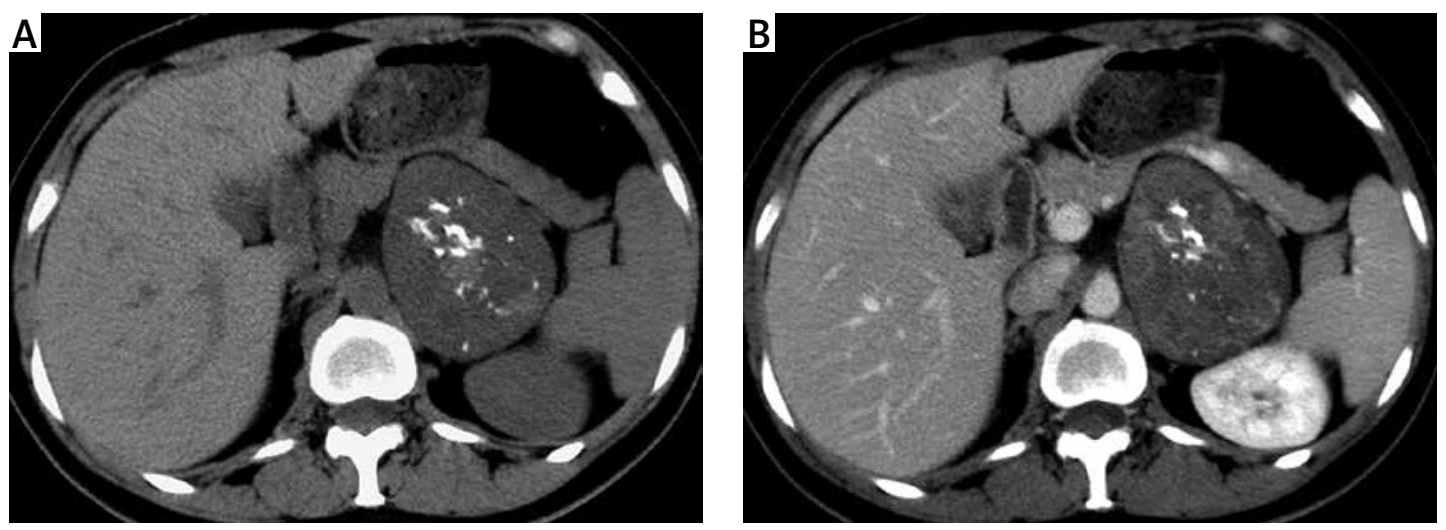

Figure 1. Unenhanced (A) and contrast-enhanced CT (B) scan shows a well-defined soft tissue mass arising from the left adrenal gland, and dot-like calcifications in the mass are revealed

adrenal schwannoma. Her catecholamine level normalized immediately after the operation. Nineteen months after the excision, no recurrence or metastases have been reported.

Schwannoma is a neural sheath tumor arising from the Schwann cell or nerve-cell sheath. Although the tumor can involve any place where Schwann cells are present, involvement of the adrenals is uncommon [1-4]. Histologically, most adrenal neoplasms arise from constituent cortical cells, but adrenal schwannomas are thought to originate from nerves in the adrenal medulla. The hypothesis is supported because the adrenal medulla is innerved by two groups of myelinated nerve fibers: one is derived from the sympathetic trunk or vagus nerve; another is from the phrenic nerve. The tumors are usually benign and slow-growing, but rarely may be malignant. Most malignant neurilemmomas are associated with von Recklinghausen's disease (VRD) or other types of neurofibromatosis [2, 3].

Clinically, adrenal schwannoma is usually asymptomatic, but some patients can present with either abdominal or flank pain. Laboratory studies are typically unremarkable. When adrenal tumors secrete catecholamines, pheochromocytomas and

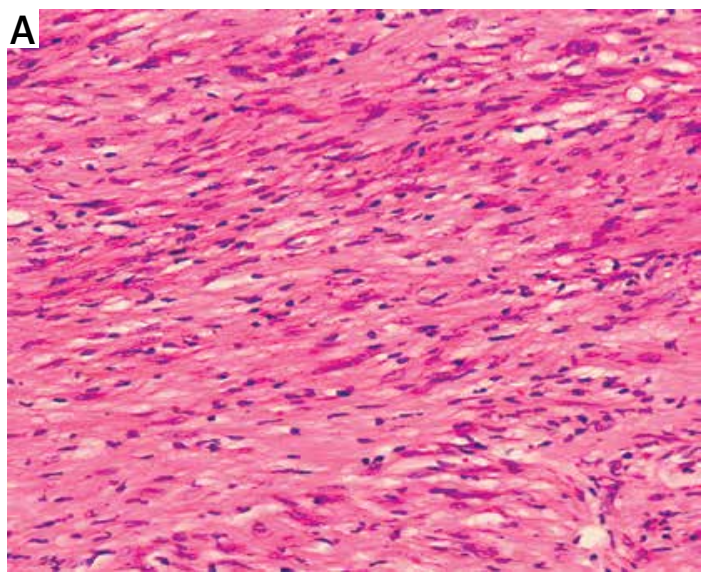

paragangliomas should be considered for differential diagnosis. Pheochromocytomas and paragangliomas are tumors arising from sympathetic and parasympathetic paraganglia, which can be located in the adrenal medulla or in extra-adrenal abdominal, intrapelvic, or thoracic locations [10, 11]. Additionally, adrenal medulla hyperplasia may produce symptoms similar to those of pheochromocytoma. Although schwannomas are usually nonsecreting tumors, those tumors may occasionally have endocrine function. So far, to the best of our knowledge, only one case of retroperitoneal schwannoma with endocrine function has been reported in the literature. Hori et al. [7] reported a 66-year-old woman with asymptomatic retroperitoneal schwannoma, but her serum noradrenalin and vanillylmandelic acid levels were increased remarkably. Our case is extremely rare owing to the adrenal schwannoma with serum adrenaline and norepinephrine overproduction confirmed by laboratory examination.

Imaging plays a key role in the diagnosis and evaluation of an adrenal mass. Although some patients may present with clinical symptoms secondary to the mass effect of tumor, most of them are discovered incidentally by imaging examina-

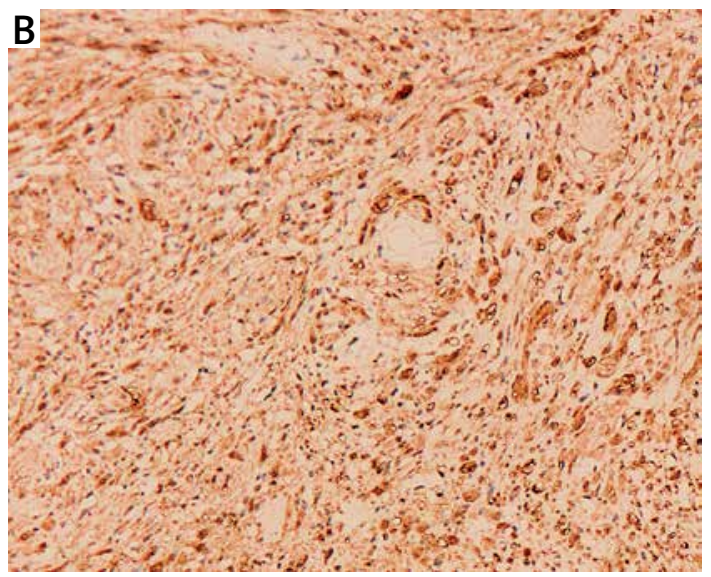

Figure 2. Microscopic examination shows the tumor exhibit Antoni A areas composed of well-defined fascicles of spindle cells and Antoni B areas (original magnification, 120; hematoxylin and eosin stain) (A). Immunohistologically, the tumor cells are positive for S-100 protein (immunohistochemical staining, 100) (B) 
tion [12]. On computed tomography, adrenal schwannoma usually present a homogeneous, soft-tissue density and well-defined mass with a surrounding fibrous capsule. The magnetic resonance imaging (MRI) scan demonstrates a hyperintense or isointense lesion on T1-weighted images, and hypointense mass on T2-weighted images. Cystic degeneration and calcifications were usually detected on CT/MRI images. According to the previous literature, cystic degeneration can be detected on images in three-fourths of retroperitoneal malignant schwannomas [3-5]. In our case, CT images showed heterogeneous density of the mass with dot-like calcification. After the administration of intravenous contrast medium, adrenal schwannoma may demonstrate variable homogeneous or heterogeneous enhancement. The CT scan provides useful information concerning the anatomic location, morphologic features, and relationships to surrounding structures. When large masses are found in retroperitoneal regions, two-dimensional multiplanar and three-dimensional reconstruction images provide an important method to evaluate the mass and the involvement of the vital adjacent neurovascular structures, and offer the potential to improve diagnostic confidence and accuracy so as to avoid unnecessary biopsy. When the pheochromocytomas/paragangliomas with catecholamine hypersecretion need be differentiated, ${ }^{18} \mathrm{~F}$-DOPA PET and ${ }^{123}$-MIGB are sensitive and specific imaging modalities for the detection and staging of these entities in various genotypes [11]. Recently, a minimally invasive approach using laparoscopic adrenalectomy was recommended, and CT may be helpful for establishing a surgical operation plan to excise the lesion adequately [8]. The preoperative diagnosis of primary adrenal schwannoma cannot be made based on imaging alone, and the definitive diagnosis depends on histological and immunohistochemical examinations, especially tumor cells being positive to S-100 protein $[2,9]$.

In conclusion, the current study demonstrates, for the first time, that a case of adrenal schwannoma with catecholamine overproduction is an extremely rare retroperitoneal neoplasm. Clinical and morphologic diagnosis of primary adrenal schwannoma may be difficult. Although extremely rare, schwannoma should be considered in the differential diagnosis of primary adrenal lesions, especially in patients with catecholamine hypersecretion.

\section{Acknowledgments}

Jianglong $\mathrm{Hou}$ and Lizhi Zhang contributed equally to this work and should be considered cofirst authors.

\section{Conflict of interest}

The authors declare no conflict of interest.

\section{References}

1. Mohiuddin Y, Gilliland MG. Adrenal schwannoma: a rare type of adrenal incidentaloma. Arch Pathol Lab Med 2013; 137: 1009-14.

2. Lau SK, Spagnolo DV, Weiss LM. Schwannoma of the adrenal gland: report of two cases. Am J Surg Pathol 2006; 30: 630-4.

3. Suzuki K, Nakanishi A, Kurosaki Y, Nogaki J, Takaba E. Adrenal schwannoma: CT and MRI findings. Radiat Med 2007; 25: 299-302.

4. Inokuchi T, Takiuchi H, Moriwaki Y, et al. Retroperitoneal ancient schwannoma presenting as an adrenal incidentaloma: CT and MR findings. Magn Reson Imaging 2006; 24: 1389-93.

5. Arena V, DeGiorgio F, Drapeau CM, Monego G, De Mercurio D, Capelli A. Adrenal schwannoma: report of two cases. Folia Neuropathol 2004; 42: 177-9.

6. Korets R, Berkenblit R, Ghavamian R. Incidentally discovered adrenal schwannoma. JSLS 2007; 11: 113-5.

7. Hori T, Yamagiwa K, Yagi S, et al. Noradrenalin-secreting retroperitoneal schwannoma resected by hand-assisted laparoscopic surgery: report of a case. Surg Today 2006; 36: 1108-13.

8. Onoda N, Ishikawa T, Toyokawa T, Takashima T, Wakasa K, Hirakawa K. Adrenal schwannoma treated with laparoscopic surgery. JSLS 2008; 12: 420-5.

9. Xiao C, Xu B, Ye H, Yang Q, Wang L, Sun YH. Experience with adrenal schwannoma in a Chinese population of six patients. J Endocrinol Invest 2011; 34: 417-21.

10. Pacak KI, Eisenhofer G, Ilias I. Diagnosis of pheochromocytoma with special emphasis on MEN2 syndrome. Hormones (Athens) 2009; 8: 111-6.

11. Rischke HC, Benz MR, Wild D, et al. Correlation of the genotype of paragangliomas and pheochromocytomas with their metabolic phenotype on 3,4-dihydroxy-6-18F-fluoro-L-phenylalanin PET. J Nucl Med 2012; 53:1352-8.

12. Pawlikowski $M$, Jaranowska $M$, Pisarek $H$, Kubiak $R$, Fuss-Chmielewska J, Winczyk K. Ectopic expression of follicle-stimulating hormone receptors in thyroid tumors. Arch Med Sci 2015; 11: 1314-7. 